\title{
Structure et dynamique de la flore et la végétation de la réserve spéciale botanique de Noflaye (Sénégal)
}

\author{
Jules DIOUF*, Mame Samba MBAYE, Abdoul Aziz CAMARA, Birane DIENG, \\ Ndongo DIOUF, Maniane SARR et Kandioura NOBA
}
Laboratoire de Botanique Biodiversité, Département de Biologie Végétales, Faculté des Sciences et Techniques, Université Cheikh Anta DIOP, B.P.5005 Dakar-Fann, Sénégal.
*Auteur correspondant; E-mail : dioufjules39@yahoo.com, jules.diouf@ucad.edu.sn ;

$$
\text { Tel: (+221) } 777974424
$$

\section{REMERCIEMENTS}

Les auteurs de ce travail remercient le personnel du laboratoire de Botanique-Biodiversité et de l'Herbier Dakar de l'Université Cheikh Anta Diop de Dakar, la Direction des Eaux, Forets, Chasses et de la Conservation des Sols (DEFCCS) et la Société d'Etude et de Protection des Tortues en France et dans le Monde (SOPTOM) pour la mise en disposition de la Réserve.

\section{RESUME}

La réserve spéciale botanique de Noflaye est un des rares écosystèmes particuliers de la zone des niayes de Dakar de par sa diversité floristique. Ce complexe est aujourd'hui menacé par un certain nombre de facteur tel que l'urbanisation. Cette étude a été réalisée pour caractériser la structure de la végétation du milieu. L'utilisation de la méthode des relevés dendrométriques nous a permis de caractériser la végétation du milieu. Les résultats indiquent que la richesse floristique du milieu est de 65 espèces ligneuses réparties en 50 genres et 31 familles avec une dominance des espèces soudanienne (39\%). L'analyse de la contribution spécifique montre que Lepisanthes senegalensis (19,7\%), Neocarya macrophylla $(14,5 \%)$ et Grewia bicolor $(13,4 \%)$ sont les plus fréquentes dans le milieu. Les valeurs élevées de densité théorique, de densité réelle, de surface terrière et de recouvrement aérien montrent que le milieu est dominé par des espèces ligneuses de petit diamètre (5 à 10 $\mathrm{cm})$ à cime jointive. La régénération est dominée par Boscia senegalensis (53\%). Ces résultats obtenus sont importants dans la conservation du milieu et la mise en place d'un plan de gestion.

(C) 2019 International Formulae Group. All rights reserved.

Mots clés : Flore, végétation, réserve spéciale botanique, Noflaye, Sénégal.

\section{Structure and dynamics of the flora and vegetation of the botanical reserve Noflaye (Senegal)}

\begin{abstract}
The botanical special reserve of Noflaye is one of the rare special ecosystems of the zone of the niayes of Dakar because of its floristic diversity. This complex is now threatened by a number of factors such as urbanization. This study was carried out to characterize the structure of the vegetation of the environment. The use of the dendrometric survey method allowed us to characterize the vegetation of the environment. The
\end{abstract}


results indicate that the floristic richness of the environment is 65 woody species divided into 50 genera and 31 families with Sudanese species dominance (39\%). The analysis of the specific contribution shows that Lepisanthes senegalensis (19.7\%), Neocarya macrophylla (14.5\%) and Grewia bicolor (13.4\%) are the most common in the environment. The high values of theoretical density, true density, basal area and overlying cover show that the medium is dominated by woody species of small diameter $(5$ to $10 \mathrm{~cm})$ with contiguous crowns. Regeneration is dominated by Boscia senegalensis (53\%). These results are important in environmental conservation and the implementation of a management plan.

(C) 2019 International Formulae Group. All rights reserved.

Keywords: Flora, vegetation, botanical special reserve, Noflaye, Senegal.

\section{INTRODUCTION}

Les ressources forestières constituent une composante majeure de l'environnement. Maillons essentiels de la chaine de la vie, elles jouent un rôle capital dans le cycle de l'eau, la régulation du climat et l'exploitation des bois pour divers usages. Elles constituent donc un enjeu d'avenir pour les pays par leur potentiel écologique et social (MEPN, 2006). Situé à l'extrême Ouest du continent africain, le Sénégal se trouve dans la zone de transition entre le désert du Sahara au Nord et la forêt humide tropicale au Sud. Le pays dispose ainsi d'un potentiel riche en diversité biologique qui se reflète au travers la diversité de ses écosystèmes (MEPN, 2006).

Les Niayes, écosystème particulier, représentent une bande de terre située le long du littoral nord entre Dakar et Saint Louis. Ces milieux sont des dépressions hydromorphes inondées par des fluctuations de la nappe phréatique au cours de l'année. Cet écosystème constitue une zone humide côtière située en plein sahel et présente une végétation azonale avec des espèces soudanoguinéennes (Prosopis africana, Kigelia africana, Antiaris africana, Detarium senegalensis, Parkia biglobosa, Elaeis guineensis). Cette végétation est actuellement très dégradée à cause de plusieurs facteurs parmi lesquels la sécheresse (MEPN, 2006) et la pression foncière. Les actions anthropiques sont responsables des changements les plus rapides des communautés végétales, même si le facteur climatique est le plus déterminant dans la distribution des espèces (Tremblay et al., 2002 ; Dessey, 2006 ; Diop, 2019). La fragmentation des habitats est actuellement l'une des menaces majeures pour la biodiversité à l'échelle globale (Yeo et al., 2013).

Ainsi dans le but de protéger une partie de cet écosystème particulier, une réserve spéciale botanique de 15,9 ha a été créée en 1957 à proximité du village de Noflaye. Cette réserve a fait l'objet d'un suivi et de plusieurs études botaniques en 1957 et en 1992 qui ont révélé que ce milieu présente un immense intérêt sur le plan floristique (Sarr, 2018). Cette zone dont la biodiversité est entretenue par des facteurs climatiques et géomorphologiques favorables, est aujourd'hui en voie de dégradation avancée à cause d'une urbanisation poussée. Selon Diallo et al. (2009), la zone des niayes est menacée de disparition sous l'effet d'une urbanisation accélérée, particulièrement dans la région de Dakar. Face aux enjeux de construction d'un environnement de proximité convenable pour l'homme en ville (Osseni et al., 2014), la sauvegarde de ces écosystèmes particuliers passe nécessairement par une connaissance de leur flore et de leur végétation (Bassène et al., 2014).

C'est dans cette optique que ce travail est entrepris pour contribuer à la connaissance de la dynamique évolutive de la végétation de la réserve spéciale de Noflaye. Il se propose de caractériser la flore et la végétation ligneuses de la réserve.

\section{MATERIEL ET METHODES \\ Présentation de la zone d'étude}

La "réserve" spéciale botanique de Noflaye est située dans la Région de Dakar entre $17^{\circ} 20^{\prime}$ de longitude Ouest et $14^{\circ} 48^{\prime}$ de latitude Nord (Figure 1). Elle est localisée à 
$40 \mathrm{~km}$ au Nord-Est de Dakar, dans le département de Rufisque et fait partie de la commune de Sangalkam.

\section{Méthode de collecte}

La méthodologie utilisée pour l'étude de la flore et de la végétation ligneuse a été conçue à partir d'informations recueillies dans la documentation relative à l'inventaire forestier. Une carte d'occupation du sol a été à la base de notre échantillonnage. Cette carte d'occupation des terres a subi un maillage. L'outil fishnet de Arc toolbox a été utilisé pour définir des mailles et les centres de chaque maille sont donnés par le logiciel qui nous a permis d'extraire les coordonnées de tous les centres de maille. La grille utilisée est constituée de placette carrée de $50 \mathrm{~m}$ x $50 \mathrm{~m}$ et un total de 69 mailles ont été délimitées dans les 15,9 ha de la réserve (Figure 2). Ainsi, un nombre total de 34 mailles a été ciblée. Une maille est dite caractéristique si elle est entièrement incluse dans l'unité d'occupation des terres c'est-à-dire homogène. Les mailles dont la moitié est en dehors de la réserve sont exclues.

Des mesures ont été effectuées sur les arbres, arbustes et lianes dont le diamètre de pré comptage est supérieur ou égal à $5 \mathrm{~cm}$ à $0,3 \mathrm{~m}$ du sol. Seuls les sujets dont le diamètre est inférieur à $5 \mathrm{~cm}$ ont été pris en compte dans la régénération.

Les variables suivantes ont été mesurées :

a le diamètre à hauteur de poitrine (DHP) ;

a la hauteur de la plus haute tige ;

a le diamètre du houppier (N-S et E-O) ;

a la distance entre les arbres.

L'identification et la détermination des espèces ont été réalisées à l'aide :

A) de flores (Berhaut 1971, 1974, 1975, 1976, 1979; Hutchinson et al., 1954, 1958, 1963, 1968, 1972) ;

a de manuels (Arbonnier, 2002);

La nomenclature employée suit la base de données gérée au Conservatoire et Jardin Botanique (CJB) de Genève; cette base de données est basée sur les importants travaux de Lebrun et Stork (1991, 1992, 1995, 1997).

La classification utilisée est celle d'APG III (2009).

\section{Traitement des données}

L'analyse de la végétation cherchait à :

- Au plan qualitatif,

के les principaux groupements ont été identifiés ;

a) les corrélations entre espèces, groupements d'espèces, facteurs naturels du milieu et facteurs anthropiques ont été établis.

a) Au plan quantitatif,

a le degré de présence a été déterminé;

فे la quantité moyenne et le coefficient de recouvrement ont été déterminés;

$\vec{a} \quad$ le point sur la fréquence spécifique a été fait;

a) la richesse spécifique (par unité de surface) a été évaluée.

Les données dendrométriques ont permis de calculer les paramètres de caractérisation de la végétation. Ainsi, les paramètres pertinents sont présentés cidessous.

La densité réelle qui est le nombre d'individus par unité de surface (individus.ha ${ }^{1}$ ) a été déterminée par la formule suivante ;

$$
\boldsymbol{D} \boldsymbol{r}=\frac{\boldsymbol{N} \boldsymbol{i}}{\boldsymbol{S}}
$$

$\mathrm{Dr}=$ Densité réelle en ind/ha ;

$\mathrm{Ni}=$ Nombre d'individu dans l'échantillon considéré ; $\mathrm{S}=$ surface de l'échantillon en ha.

La densité théorique qui peut être exprimée par l'écartement moyen entre les individus en utilisant la méthode du plus proche individu. Son calcul se fait par le rapport de la surface d'un hectare $\left(10000 \mathrm{~m}^{2}\right)$ sur le carré de la distance moyenne entres les arbres (dm) (Wouters et Notelaers, 1999).

$$
D_{t h}=\left(\frac{100}{d m}\right)^{2}
$$

Avec $\boldsymbol{D}_{t h .}=$ Densité théorique en ind/ha $; \boldsymbol{d}_{\boldsymbol{m}}=$ distance moyenne en mètre $(\mathrm{m})$.

La surface terrière (St) ou recouvrement basal correspond à la surface transversale du tronc du DHP. C'est la somme des surfaces terrières de tous les individus dont le DHP est supérieur ou égal à $5 \mathrm{~cm}$. Elle désigne la 
proportion de la surface d'un échantillon occupée par l'aire d'ancrage (évaluée à la base) d'un individu, d'une espèce ou d'un peuplement. Elle permet de juger l'état et de la dynamique d'une population ou d'une essence à partir de son DBH (Gning, 2008 ; Ngom et al., 2013). Pour un individu, c'est le rapport entre la surface de la section transversale de son tronc à $1,3 \mathrm{~m}$ du sol et la surface de l'échantillon considérée. Elle est obtenue à partir de la formule suivante :

$$
S_{t}=\frac{\pi\left(\frac{d_{1,3}}{2}\right)^{2}}{S_{E}}
$$

Avec $\boldsymbol{S}_{\boldsymbol{t}}=$ surface terrière ; $\boldsymbol{d}_{\mathbf{1 , 3}}=$ diamètre en $\mathrm{m}$ du tronc à $0,3 \mathrm{~m}$ du sol; $\boldsymbol{S}_{\boldsymbol{E}}=$ surface de l'échantillon considéré en ha.

Le recouvrement aérien correspond à la proportion de la surface d'un échantillon occupée par la projection verticale au sol de la couronne d'un végétal $\left(\mathrm{m}^{2} \cdot \mathrm{ha}^{-1}\right)$. Tel que décrit par Ngom et al. (2013), c'est la somme des surfaces de la couronne de tous les individus du peuplement.

$$
G=\frac{\pi\left(\frac{d_{m h}}{2}\right)^{2}}{S_{E}}
$$

où $\boldsymbol{G}=$ recouvrement aérien $; \boldsymbol{d}_{\boldsymbol{m} h}=$ diamètre moyen du houppier en $\mathrm{m}$, qui est égal à la moitié de la somme des diamètres Nord-Sud et Est-Ouest ; $\boldsymbol{S}_{\boldsymbol{E}}=$ surface de l'échantillon considéré en ha.

Indice de diversité de Shannon $(H)$ :

$H=-\sum_{i=1}^{S} p_{i} \cdot \log _{2}\left(p_{i}\right)$

avec

$\mathrm{pi}=$ abondance proportionnelle ou pourcentage d'importance de l'espèce, $\mathrm{pi}=\mathrm{ni} / \mathrm{N}, \mathrm{S}=$ Nombre total d'espèce ; $\mathrm{ni}=$ nombres d'individus d'une espèce dans l'échantillon; $\mathrm{N}=$ nombres total d'individus de toutes les espèces dans l'échantillon (Gray et al., 1992).

Indice d'équitabilité de Piélou (J) : $\mathrm{J}=\mathbf{H} / \mathbf{H}_{\text {max }}$

$$
H_{\text {max }}=\log _{2}(S)
$$

avec $\mathrm{S}=$ nombre total d'espèce

L'indice de diversité de Shannon et celui d'équitabilité, sont deux mesures de la diversité spécifique parmi les plus connues. L'indice de Shannon est minimal si tous les individus appartiennent à la même espèce et maximal quand tous les individus sont répartis de façon égale sur toutes les espèces (Da Sié, 2009).

L'appréciation de la régularité de la distribution des individus a été faite par l'indice d'équitabilité de Piélou (Blondel, 1979). Cet indice varie de 0 (dominance d'une espèce) à 1 (équirépartition des individus dans les espèces). Il est maximal quand les espèces ont des abondances identiques dans le peuplement et minimal quand une seule espèce domine le peuplement.

Analyse de la structure et de la dynamique de la végétation ligneuse

La structure de la végétation et des populations des espèces ligneuses a été établie sur la base d'une distribution des individus dans des classes de diamètre et de hauteur. Pour ce faire, onze classes d'amplitude $5 \mathrm{~cm}$ ont été définies pour les DHP et six classes d'amplitude $2 \mathrm{~m}$ pour les hauteurs.

L'analyse de la régénération a été faite en adoptant deux classes. Une première comprenant des individus dont la hauteur n'atteint pas les $1,3 \mathrm{~m}$ et la deuxième comprend les individus dont la hauteur dépasse les 1,3 m mais dont le diamètre de pré comptage n'est pas atteint. Le taux et l'importance spécifique de régénération (ISR) ont été déterminés.

Le taux de régénération du peuplement (TRP) est donné par le rapport en pourcentage entre l'effectif total des jeunes plants et l'effectif total du peuplement.

TRP $=\frac{\text { Effectif totaldes jeunes plants }}{\text { Effectif totaldu peuplement }} x 100$

L'importance spécifique de régénération (ISR) est obtenue à partir du rapport entre l'effectif des jeunes plants d'une espèce et l'effectif de jeunes plants dénombrés.

$$
I S R=\frac{\text { Effectif des jeunes plants d' une espèces }}{\text { Effectif total des jeunes plants dénombrés }} \times 100
$$




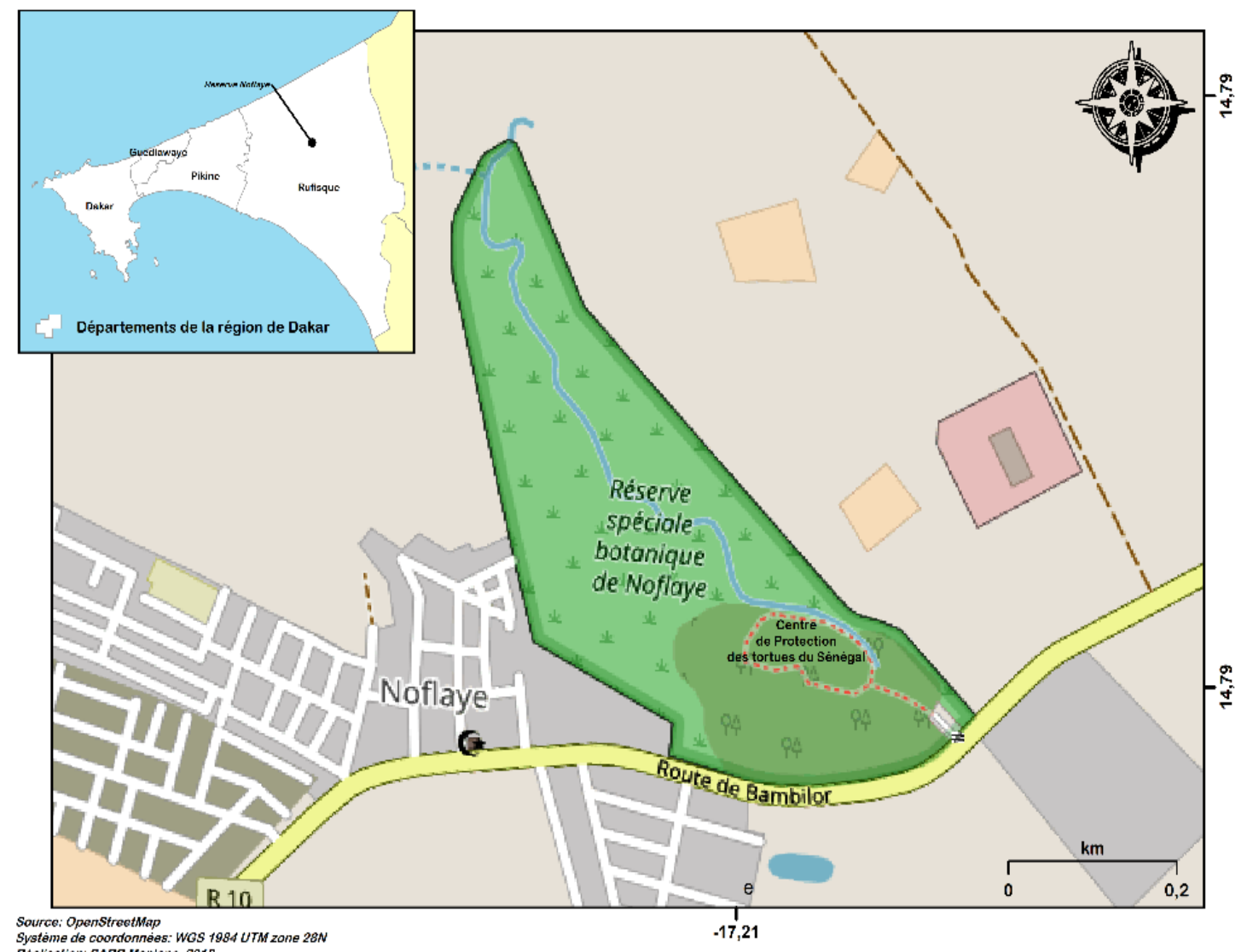

Figure 1 : Localisation de la réserve spéciale botanique de Noflaye.

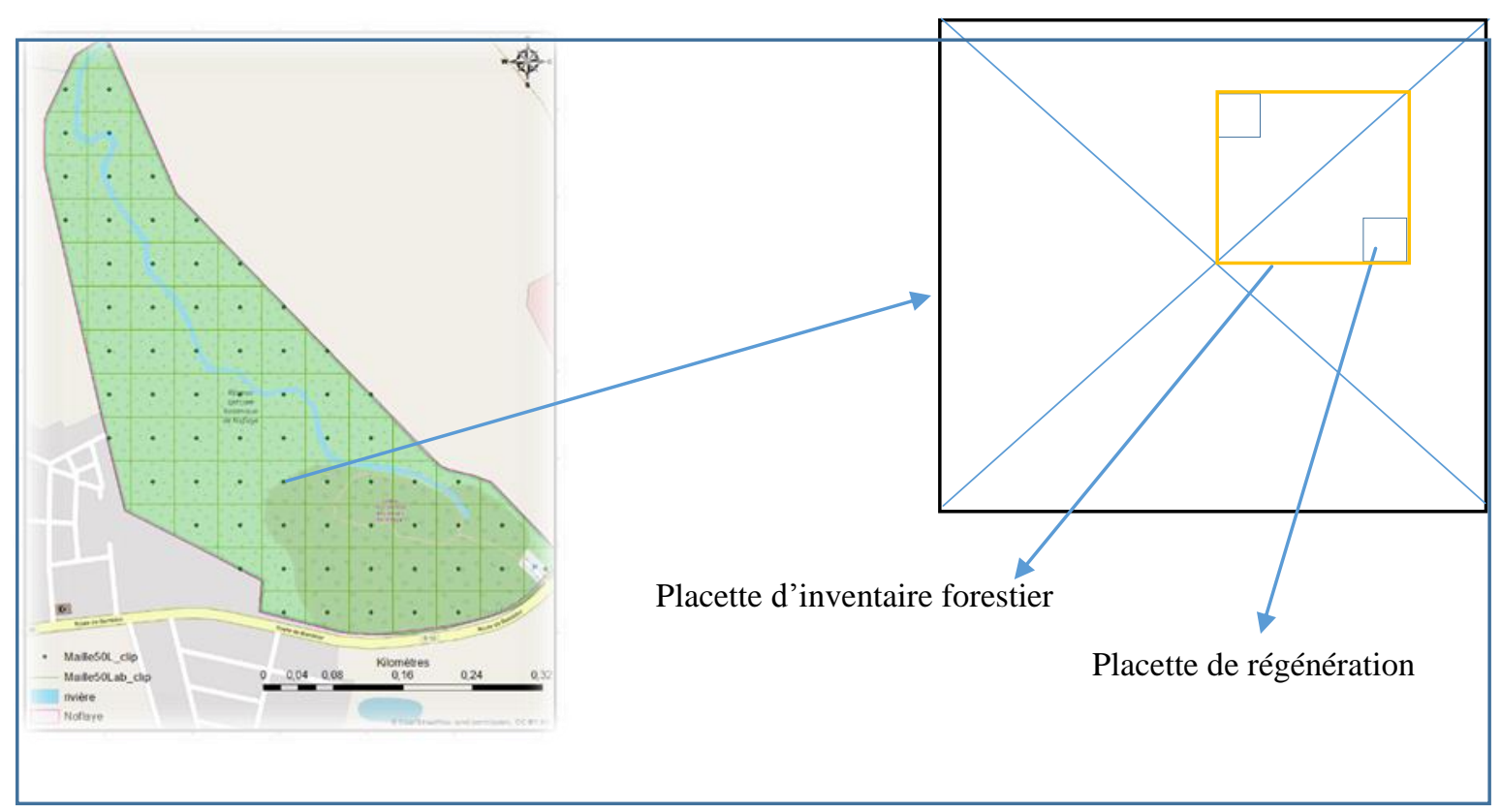

Figure 2 : Dispositif d'échantillonnage pour l'inventaire forestier. 


\section{RESULTATS}

Le Tableau 1 présente la liste globale des espèces ligneuses et familles rencontrées au cours de l'inventaire forestier dans la Réserve Spéciale Botanique de Noflaye. Pour chaque espèce, le nom scientifique, la famille et l'affinité phytogéographique sont donnés.

La richesse spécifique totale de la RSBN est de 65 espèces. Ces espèces sont réparties en 50 genres et 31 familles. Les familles les plus représentées sont les familles des Fabaceae (14 espèces) suivies de celles des Moraceae (7 espèces), des Apocynaceae et des Malvaceae avec chacune 5 espèces. Les espèces à affinités soudaniennes sont prédominantes dans le milieu avec 39\% de l'effectif total. Elles sont suivies par les espèces soudanoguinéennes et sahélo-soudaniennes avec respectivement $15,6 \%$ et $14,1 \%$. En revanche, les espèces à affinités guinéo-soudaniennes représentent $10,9 \%$ contre $7,8 \%$ pour les espèces sahéliennes. Quant aux espèces guinéennes, elles représentent $1,6 \%$, et sont donc très faiblement représentées dans le complexe de Noflaye.

\section{Caractérisation de la végétation ligneuse}

Les paramètres structuraux de la réserve spéciale botanique de Noflaye sont consignés dans le tableau 2 . La densité réelle de la réserve spéciale botanique de Noflaye est de 334,5 ind.ha- ${ }^{1}$. Six espèces parmi les 65 espèces ligneuses sont relativement bien représentées: Lepisanthes senegalensis $(19,7 \%)$, Neocarya macrophylla (14,5\%), Grewia bicolor (13,4\%), Acacia ataxacantha $(8,9 \%)$, Crataeva adansonii $(8,6 \%)$ et Maytenus senegalensis (7,1\%). La densité théorique est de 1101,00 ind.ha- ${ }^{1}$. La surface terrière est de $4,00 \mathrm{~m}^{2} \cdot \mathrm{ha}^{-1}$. Certaines espèces apparaissent nettement dominantes. Ces espèces sont: Ficus lutea (19,33\%), Adansonia digitata (16,14\%), Lepisanthes senegalensis (15,21\%), Grewia bicolor $(10,21 \%)$, Neocarya macrophylla $(8,97 \%)$. L'aire de projection verticale de cette réserve est de $3025,79 \mathrm{~m}^{2}$.ha ${ }^{-1}$. Trois espèces sont plus représentatives en termes de recouvrement. Il s'agit de Ficus lutea (22,22\%), Lepisanthes senegalensis $(17,42 \%)$ et Grewia bicolor $(13,51 \%)$. Le taux de régénération du peuplement est de 45,22\% correspondant à un peuplement vieillissant avec une densité des pieds âgés supérieure à celle des jeunes plants. La diversité biologique du milieu a été appréciée par l'indice de Shannon et d'équitabilité. L'indice de Shannon est de 1,19 montrant une diversité assez importante dans le milieu. L'appréciation de la régularité de la distribution des individus a été faite par l'indice d'équitabilité de Piélou. Cet indice est de 0,48 montrant ainsi que la distribution des individus dans le milieu est appréciable malgré une abondance relative d'individus de Lepisanthes senegalensis, Neocarya macrophylla, Grewia bicolor, Acacia ataxacantha, Crataeva adansonii et de Maytenus senegalensis.

\section{Contribution spécifique (CSP)}

La Figure 3 regroupe des informations sur la contribution spécifique dans la Réserve Spéciale Botanique de Noflaye (RSBN). L'analyse de cette Figure 3 a permis de regrouper les espèces ligneuses de ce complexe forestier de Noflaye en trois catégories.

- Un premier groupe fortement représenté occupant plus de $40 \%$ de l'effectif total $\mathrm{du}$ milieu. Les espèces de ce groupe sont Lepisanthes senegalensis (19,7\%), Neocarya macrophylla $(14,5 \%)$ et Grewia bicolor $(13,4 \%)$. Elles sont respectivement à affinités soudanienne, soudano-guinéenne et sahélosoudanienne.

- Le second groupe est constitué d'espèces moyennement représentées et occupe $24,6 \%$ de l'effectif total. Deux de ces espèces à savoir Acacia ataxacantha $(8,9 \%)$ et Maytenus senegalensis $(7,1 \%)$ sont des espèces épineuses donc ayant une forte capacité de résistance. Crataeva adansonii (8,6\%) est une espèce qui se multiplie par drageonnage ce qui lui confère sa forte capacité de résistance.

- Le troisième groupe qui constitue le groupe des espèces faiblement représenté est le plus diversifié en termes d'espèces et occupe plus de $20 \%$ de l'effectif total. 
Dans ce groupe, à l'exception de trois espèces guinéo-soudaniennes que sont Ficus lutea, Lonchocarpus sericeus et Morus mesozia, la plus part des espèces sont à affinités soudanienne ou sahélo soudanienne. Ce groupe est constitué d'espèces indicatrices de l'évolution du milieu.

\section{Structure globale, dynamique et tendances évolutives des populations des espèces}

Les Figures 4 et 5 donnent des informations sur les tendances évolutives de la population floristique de la Réserve Spéciale Botanique de Noflaye. L'analyse de cette structure montre que le milieu est caractérisé par une distribution des individus dans les sujets jeunes. La densité des ligneux de diamètre comprise entre 5 et 10 est relativement élevée dans la réserve. Cette structure présente une allure en forme de $\mathrm{J}$ renversé caractéristique d'un peuplement de type 1 c'est-à-dire dominé par un grand nombre de sujets jeunes, un petit nombre de grand individus avec une réduction régulière du nombre d'individus d'une classe de dimension à la suivante. Ce type de structure est caractéristique d'un peuplement stable avec une forte présence d'individus dans les classes jeunes.

A l'échelle spécifique, ce graphique montre une distribution des individus dans les classes inférieures pour le peuplement de Acacia ataxacantha, Commiphora africana, Crataeva adansonii, Grewia bicolor, Maytenus senegalensis, Khaya senegalensis et Neocarya macrophylla. La dynamique d'évolution du peuplement de ces espèces est progressive avec une forte représentation des individus dans les classes jeunes contrairement au peuplement d'Adansonia digitata dominé par des sujets de classes supérieures. Quant au Ficus lutea, la tendance évolutive de la population est stable avec une large représentativité des individus dans les classes de DBH.

\section{Caractérisation verticale de la végétation ligneuse (Hauteur)}

La distribution en classes de hauteur des individus est illustrée par des histogrammes donnant les effectifs correspondants au niveau des Figures 6 et 7 . La hauteur moyenne du peuplement est de 7,96 m. L'analyse de la figure 6 montre que le milieu est dominé par des individus dont la hauteur varie entre 2 et $8 \mathrm{~m}$. L'allure de la courbe de tendance est sous forme de cloche caractéristique d'un peuplement de type 2 dont les individus sont répartis dans les classes intermédiaires avec un effectif faible des jeunes et grands sujets. Sur le plan spécifique, l'analyse de la courbe montre une différence de structure dans la répartition des individus au sein des espèces. Parmi les 65 espèces ligneuses qui ont été récoltées dans la réserve, seules sept (7) présentent une distribution hétérogène des sujets dans les différentes classes de hauteur. Les peuplements de Acacia ataxacantha, Lepisanthes senegalensis, Crataeva adansonii, Grewia bicolor, Khaya senegalensis, Maytenus senegalensis, Neocarya macrophylla sont dominés par les individus des classes 4-6 et 6-8. Les autres espèces sont caractérisées par une distribution homogène des sujets dans les autres classes de hauteur.

Importance spécifique de régénération (ISR)

La figure 08 présente des informations sur la distribution des individus selon l'importance spécifique de régénération dans la réserve spéciale botanique de Noflaye. L'analyse de cet histogramme nous montre que Boscia senegalensis (53\%), Acacia ataxacantha $(15 \%)$ et Maytenus senegalensis (12\%) sont les trois espèces qui régénèrent le plus avec environ $80 \%$ des rejets. Cette courbe de l'importance spécifique de régénération nous montre une dominance de neuf (9) espèces qui occupent plus de $80 \%$ des sujets. D'autres espèces qui sont fortement représentées au niveau des individus adultes présentent une faible régénération. C'est le cas du Lepisanthes senegalensis (4\%), Grewia bicolor (3\%) et Crataeva adansonii (2\%). 
Tableau 1 : Liste des espèces recensées avec des indications sur les affinités phytogéographique (A.P).

\begin{tabular}{|c|c|c|c|c|}
\hline Familles & N.G & N.E & Noms scientifiques & A. $\mathbf{P}$ \\
\hline Anacardiaceae & 2 & 2 & $\begin{array}{l}\text { Anacardium occidentale L. } \\
\text { Sclerocarya birrea subsp. caffra (Sond.) } \\
\text { Kokwaro }\end{array}$ & so gu \\
\hline \multirow[t]{2}{*}{ Apocynaceae-Asclepioidae } & \multirow[t]{2}{*}{2} & \multirow[t]{3}{*}{2} & Calotropis procera (Aiton) W. T. Aiton & sa \\
\hline & & & Leptadenia lanceolata (Poir.) Goyder & so \\
\hline \multirow{3}{*}{ Apocynaceae-Apocynoidae } & \multirow{3}{*}{3} & & Baissea multiflora A. DC. & so \\
\hline & & \multirow[t]{2}{*}{3} & Saba senegalensis (A. DC.) Pichon & gu so \\
\hline & & & Strophanthus sarmentosus DC. & so \\
\hline Arecaceae & 1 & 1 & Borassus aethiopum Mart. & so \\
\hline Burseraceae & 1 & 1 & Commiphora africana (A. Rich.) Engl. & sa \\
\hline \multirow{4}{*}{ Capparaceae } & \multirow{4}{*}{3} & \multirow{4}{*}{4} & Boscia senegalensis (Pers.) Lam. ex Poir. & so sa \\
\hline & & & Cadaba farinosa Forssk. & sa so \\
\hline & & & Capparis tomentosa Lam.a & so \\
\hline & & & Crataeva adansonii DC. & so \\
\hline \multirow[t]{2}{*}{ Celastraceae } & \multirow[t]{2}{*}{2} & \multirow[t]{2}{*}{2} & $\begin{array}{l}\text { Loeseneriella africana (Willd.) N. Hallé } \\
\text { var. africana }\end{array}$ & so \\
\hline & & & Maytenus senegalensis (Lam.) Exell & so \\
\hline Chrysobalanaceae & 1 & 1 & Neocarya macrophylla (Sabine) Prance & so gu \\
\hline Combretaceae & 1 & 2 & Combretum micranthum G. Don & so \\
\hline Ebenaceae & 1 & 1 & $\begin{array}{l}\text { Combretum paniculatum Vent. } \\
\text { Diospyros mespiliformis Hochst. ex A. } \\
\text { DC. }\end{array}$ & so gu \\
\hline \multirow{4}{*}{ Euphorbiaceae } & \multirow{4}{*}{3} & \multirow{4}{*}{4} & Euphorbia balsamifera Aiton & sa \\
\hline & & & Euphorbia tirucalli L. & so \\
\hline & & & Hura crepitans L. & so sa \\
\hline & & & Jatropha gossypiifolia $\mathrm{L}$. & so sa \\
\hline \multirow[t]{2}{*}{ Fabaceae-Caesalpinoidae } & \multirow[t]{2}{*}{4} & \multirow[t]{2}{*}{4} & $\begin{array}{l}\text { Detarium senegalense J. F. Gmel. } \\
\text { Peltophorum pterocarpum (DC.) K. } \\
\text { Heyne }\end{array}$ & gu so \\
\hline & & & Piliostigma reticulatum (DC.) Hochst. & so sa \\
\hline Fabaceae-Faboidae & 1 & 1 & $\begin{array}{l}\text { Tamarindus indica } \mathrm{L} \text {. } \\
\text { Lonchocarpus sericeus (Poir.) Kunth ex } \\
\text { DC. }\end{array}$ & gu so \\
\hline \multirow{4}{*}{ Fabaceae-Mimosoidae } & \multirow{4}{*}{4} & \multirow{4}{*}{9} & Acacia ataxacantha DC. & so \\
\hline & & & Acacia mellifera (Vahl) Benth. & so \\
\hline & & & Acacia nilotica (L.) Willd. ex Delile & sa so \\
\hline & & & Acacia polyacantha Willd. & so \\
\hline
\end{tabular}




\begin{tabular}{|c|c|c|c|c|}
\hline & & & Acacia senegal (L.) Willd. & sa \\
\hline & & & Dichrostachys cinerea (L.) Wight \& Arn. & so \\
\hline & & & Faidherbia albida (Delile) A. Chev. & so sa \\
\hline & & & Prosopis africana (Guill. \& Perr.) Taub. & so \\
\hline & & & Prosopis glandulosa Torr. & so sa \\
\hline Loranthaceae & 1 & 1 & $\begin{array}{l}\text { Tapinanthus bangwensis (Engl. \& K. } \\
\text { Krause) Danser }\end{array}$ & so \\
\hline Lythraceae & 1 & 1 & Lawsonia inermis L. & so \\
\hline Malvaceae-Bombacoidae & 1 & 1 & Adansonia digitata $\mathrm{L}$. & so \\
\hline Malvaceae-Sterculoidae & 1 & 1 & Cola cordifolia (Cav.) R. Br. & so gu \\
\hline & & & Grewia bicolor Juss. & sa so \\
\hline Malvaceae-Tilicoidae & 1 & 3 & Grewia flavescens Juss. & so \\
\hline & & & Grewia villosa Willd. & sa so \\
\hline Meliaceae & 2 & 2 & Azadirachta indica A. Juss. & sa so \\
\hline & & & Khaya senegalensis (Desr.) A. Juss. & so \\
\hline & & & Ficus capensis Thunb. & gu so \\
\hline & & & Ficus lutea Vahl & gu so \\
\hline & & & Ficus polita Vahl & $\mathrm{gu}$ \\
\hline Moraceae & 3 & 7 & Ficus sycomorus L. & so gu \\
\hline & & & Ficus thonningii Blume & so gu \\
\hline & & & Morus mesozygia Stapf & gu so \\
\hline & & & $\begin{array}{l}\text { Antiaris toxicaria var. africana Scott-Elliot } \\
\text { ex A. Chev. }\end{array}$ & gu so \\
\hline Moringaceae & 1 & 1 & Moringa oleifera Lam. & so sa \\
\hline Nyctaginaceae & 1 & 1 & Bougainvillea spectabilis Willd. & so sa \\
\hline Olacaceae & 1 & 1 & Ximenia americana $\mathrm{L}$. & so sa \\
\hline Phyllantaceae & 1 & 1 & Phyllanthus reticulatus Poir. & so gu \\
\hline Rhamnaceae & 1 & 2 & Ziziphus mucronata Willd. & so gu \\
\hline & & & Ziziphus mauritiana Lam. & so \\
\hline & & & Sarcocephalus latifolius (Sm.) E. A. Bruce & so \\
\hline Rubiaceae & 2 & 2 & $\begin{array}{l}\text { Zanthoxylum zanthoxyloides (Lam.) } \\
\text { Zepern. \& Timler }\end{array}$ & so \\
\hline Salicaceae & 1 & 1 & Flacourtia indica (Burm. f.) Merr. & so \\
\hline Sapindaceae & 1 & 1 & $\begin{array}{l}\text { Lepisanthes senegalensis (Juss. ex Poir.) } \\
\text { Leenh. }\end{array}$ & so \\
\hline Tamaricaeae & 1 & 1 & Tamarix senegalensis DC. & sa \\
\hline Zygophyllaceae & 1 & 1 & Balanites aegyptiaca (L.) Delile & sa so \\
\hline TOTAL & $\mathbf{5 0}$ & 65 & & \\
\hline
\end{tabular}

A.P : Affinités Phytogéographique ; N.G : Nombre de Genres ; N.E : Nombre d'Espèce ; sa : sahélienne ; so : soudanienne; sa so : sahélo-sahélienne ; so gu : soudano-guinéenne ; so sa : soudano-sahélienne ; gu so : guinéo-soudanienne. 
Tableau 2 : Paramètres structuraux de la réserve spéciale botanique de Noflaye.

\section{Paramètres structuraux}

Densité réelle (ind. ha ${ }^{-1}$ )

Densité théorique (ind. $\mathrm{ha}^{-1}$ )

Surface terrière $\left(\mathrm{m}^{2} . \mathrm{ha}^{-1}\right)$

Recouvrement aérien $\left(\mathrm{m}^{2}\right.$. ha $\left.\mathrm{a}^{-1}\right)$

TRP $(\%)$

Indice Shannon

Indice d'équitabilité de Piélou

\section{Peuplement de Noflaye}

334,50

1101,00

4,00

3025,79

45,22

1,19

0,48

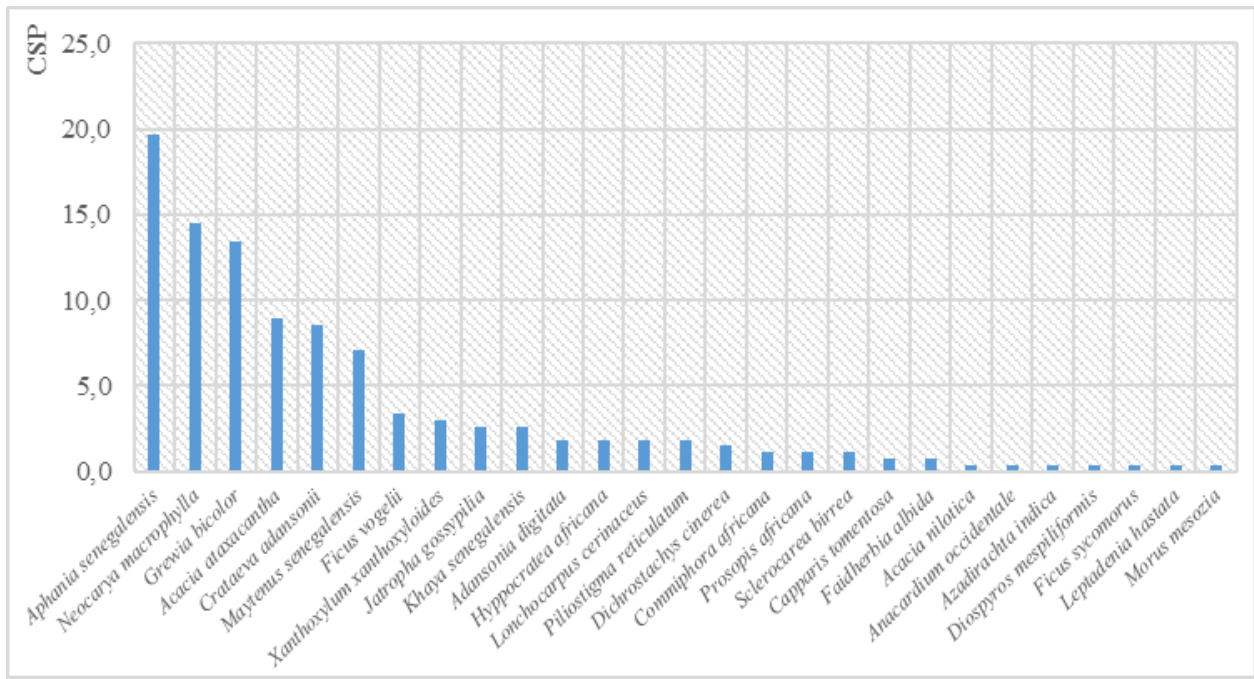

Figure 3: Contribution spécifique des espèces dans la Réserve Spéciale Botanique de Noflaye (RSBN).

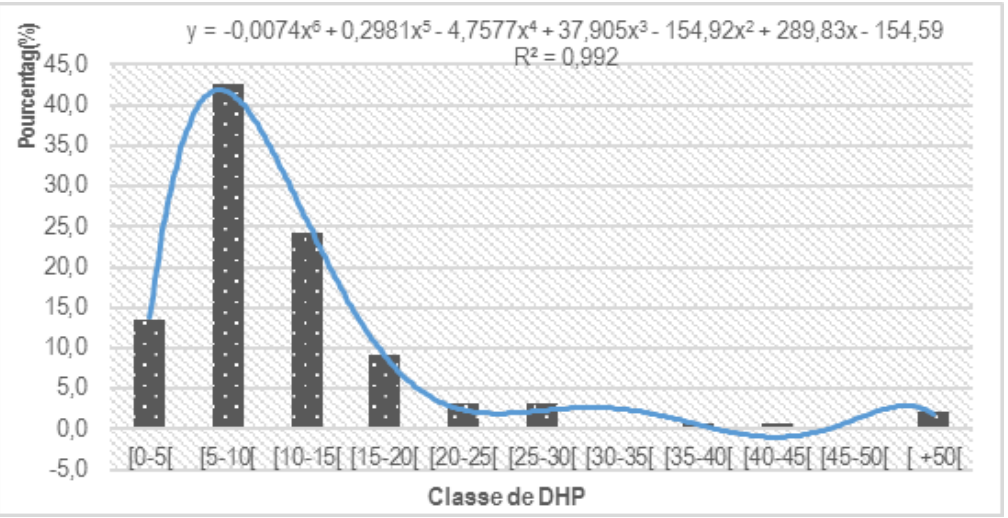

Figure 4 : Distribution des individus dans les classes de DHP. 


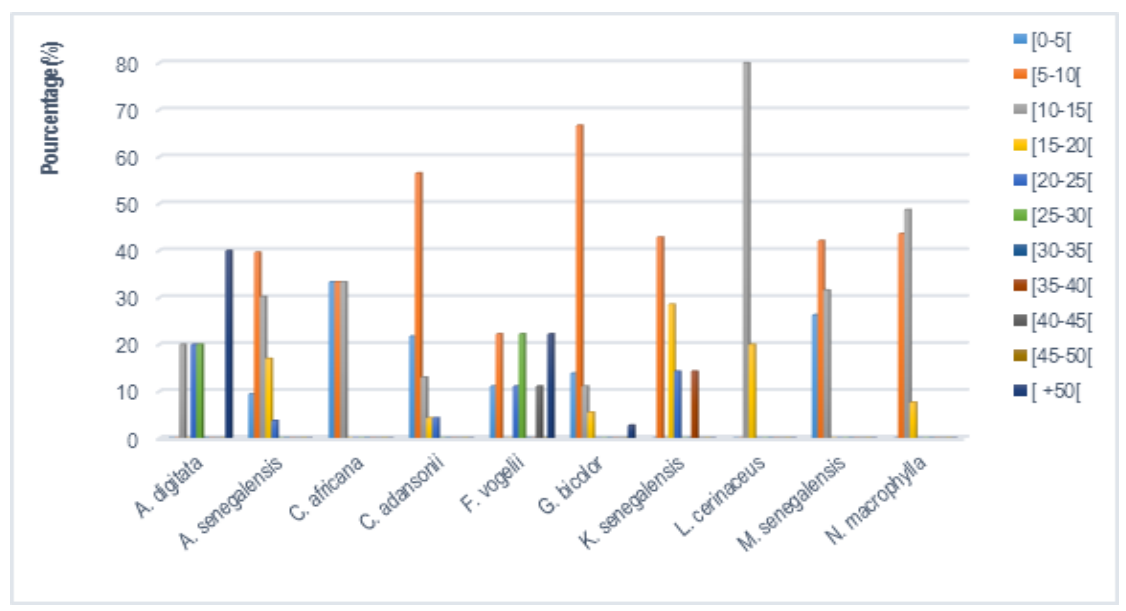

Figure 5: Distribution des espèces les plus abondantes selon les classes de DBH dans la RSBN.

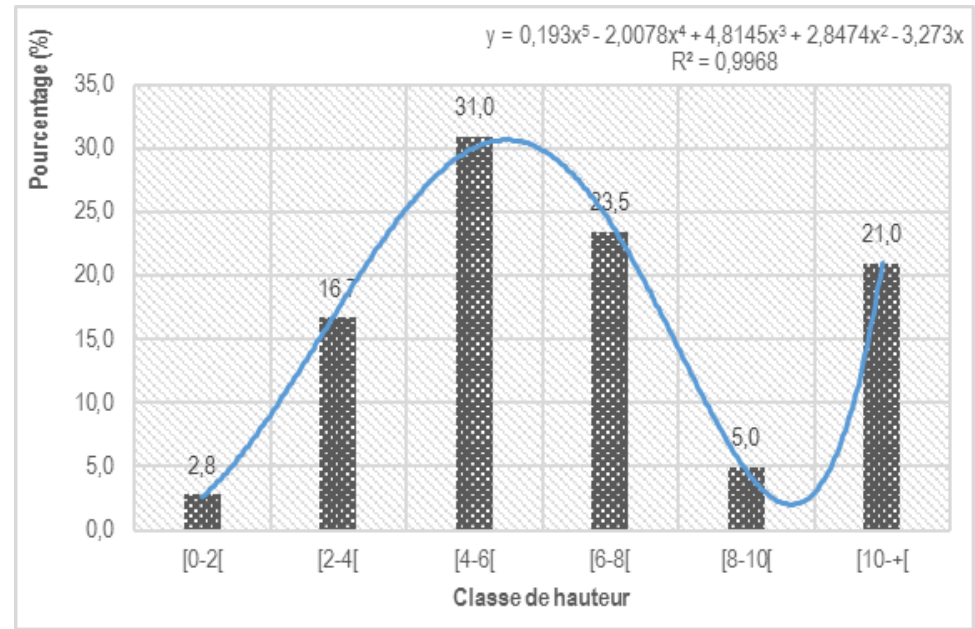

Figure 6 : Distribution des individus dans les classes de hauteur.

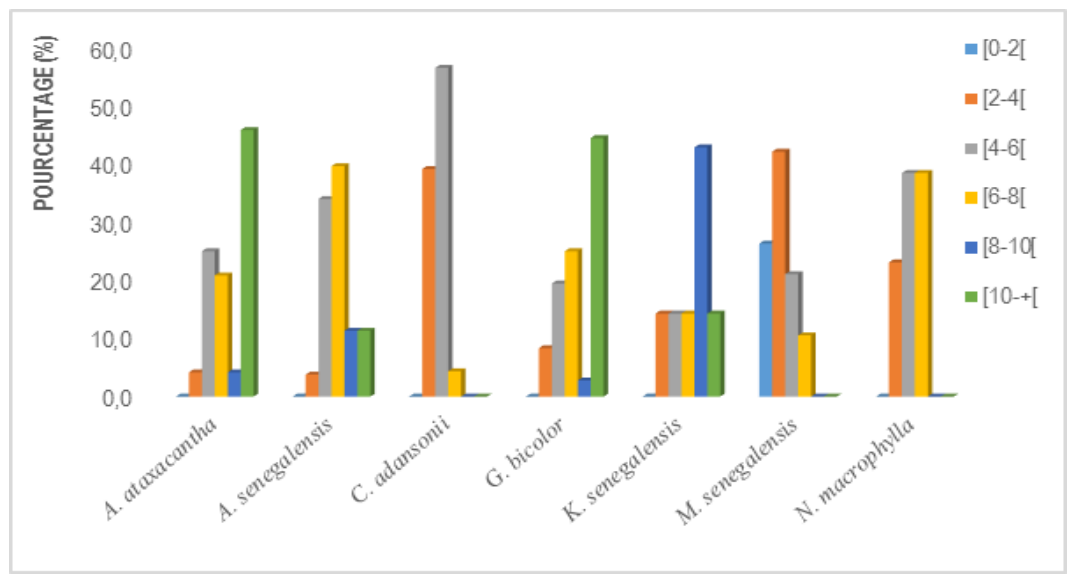

Figure 7 : Distribution des espèces les plus abondantes selon les classes de hauteurs dans la RSBN. 


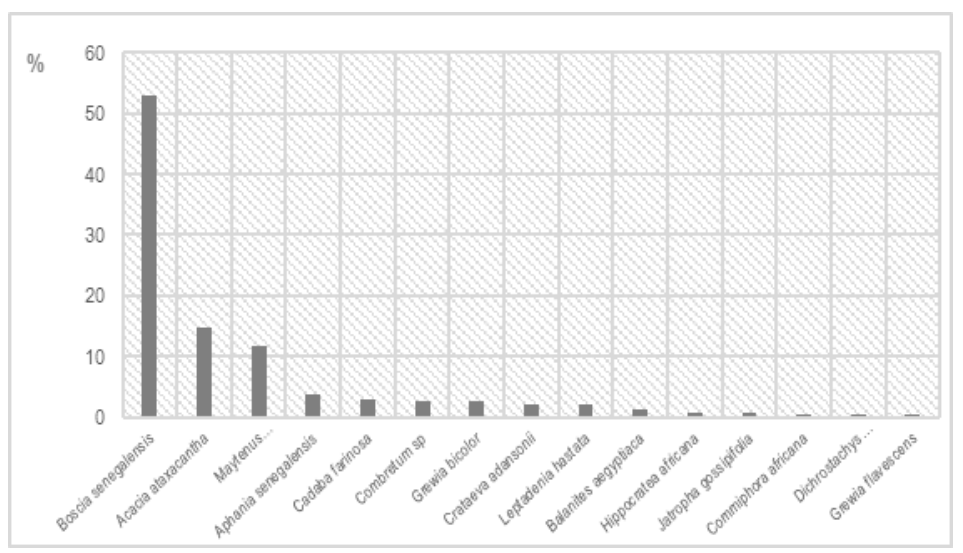

Figure 8 : Importance spécifique de régénération dans la RSBN.

\section{DISCUSSION}

La caractérisation de la flore de la réserve a révélé une richesse spécifique très importante dans le milieu. Cette flore ligneuse est dominée par des espèces à affinités soudaniennes qui occupent $39 \%$ de l'effectif du milieu. Comparée à celle de la réserve de Ngazobil, la flore ligneuse de la réserve spéciale botanique de Noflaye présente approximativement la même diversité avec 65 espèces réparties en 50 genres et 31 familles contre 60 espèces réparties en 47 genres et 25 familles pour la réserve de Ngazobil (Diatta et al., 2009). Les espèces les plus abondantes dans le milieu sont Lepisanthes senegalensis $(19,72 \%)$, Neocarya macrophylla $(14,5 \%)$ et Grewia bicolor $(13,4 \%)$ montrant ainsi que le milieu est à dominance arbustive. L'abondance de ces espèces dans la zone montre ainsi une forte progression des espèces à affinité sahélo-soudanienne au profit des espèces guinéenne qui jadis étaient caractéristiques du milieu (Diop et al., 2018). D'après ces derniers auteurs, ce phénomène pourrait s'expliquer par le déficit pluviométrique de ces dernières années mais aussi par la mise en valeur des terres par l'agriculture et l'urbanisation.

La densité réelle du peuplement est de 334,5 ind/ha. La densité théorique est trois fois supérieure à la densité réelle, ce qui traduit une distribution en agrégat des individus dans le complexe forestier avec la présence tantôt d'endroits très clairsemés ou parfois d'endroit où les sujets sont en bosquets (Gning, 2008, Ngom et al., 2013). La surface terrière est faibles $\left(4 \mathrm{~m}^{2} \cdot \mathrm{ha}^{-1}\right) \mathrm{s}$ 'expliquant par la présence d'une forte communauté de petit diamètre traduisant ainsi un peuplement à dominance arbustive. Le recouvrement aérien (3025,79 $\mathrm{m}^{2}$.ha-1) élevé dans le milieu s'explique par la présence de grands arbres à cime jointive comme Adansonia digitata, Neocarya macrophylla, Ficus vogelii, Ficus sycomorus, Khaya senegalensis, Khaya senegalensis, Morus mesozygia et Lepisanthes senegalensis.

L'indice de diversité de Shannon et d'équitabilité de Piélou sont les plus couramment utilisés pour l'évaluation de la diversité d'un milieu. Leur calcul a permis de monter que la végétation du milieu est caractérisée par la dominance des espèces présentant une faible densité. Ces valeurs reste faible par rapport à celle de la réserve naturelle de Ngazobil qui est de 1,8 (Diatta et al., 2009). Selon Abdourhamane et al. (2013), l'indice de Shannon a des valeurs fortes pour des espèces avec des recouvrements de même importance et prend des valeurs faibles lorsque quelques espèces ont de forts recouvrements. Ce dernier auteur indique que ceci pourrait être lié à un état de stress de l'écosystème.

La structure du peuplement de la RSB Noflaye selon les classes de DHP montre qu'environ $80 \%$ des sujets ont un diamètre inférieur à $20 \mathrm{~cm}$ avec une nette dominance de 
la classe comprise entre 5 et 10 . L'allure de cette courbe se présente sous forme de $\mathrm{J}$ "renversée caractéristique d'un peuplement de type1 dominé par une forte présence d'individus dans les classes jeunes. A l'échelle spécifique, la dynamique d'évolution du peuplement est progressive avec des sujets jeunes pour des espèces comme Acacia ataxacantha, Commiphora africana, Crataeva adansonii, Grewia bicolor, Maytenus senegalensis, Khaya senegalensis et Neocarya macrophylla. Selon les travaux de Abdourhamane et al., 2013, les densités élevées des sujets de petit diamètre assurent l'avenir de la formation naturelle tandis que les faibles densités de gros individus résultent de la sélection naturelle et sont en fait les semenciers qui assurent la pérennité du peuplement. Cependant la présence de certaines espèces à fort potentiel socioéconomique comme Neocarya macrophylla, Khaya senegalensis, Lepisanthes senegalensis, Sarcocephalus latifolius, Zanthoxylum zanthoxyloides, Acacia ataxacantha qui sont régulièrement utilisées par la population locale pourrait constituer une menace de conservation dans ce complexe. Sambou (2004), qui avait fait le même constat pour certaines forêts classées au Sénégal concluait qu'une analyse globale de la structure pourrait cacher un processus de dégradation qui affecte la population de certaines espèces à fort potentiel socioéconomique. La structure du peuplement ligneux de la RSBN établie suivant la hauteur montre que les classes intermédiaires regroupent plus de $70 \%$ de l'effectif de la population totale. Ces hauteurs sont comprises entre 2 et $8 \mathrm{~m}$ avec une large dominance de la classe de 4 à $6 \mathrm{~m}$. L'allure de cette courbe révèle la présence d'un peuplement à dominance arbustive clairsemée de quelques arbres.

L'importance de la densité globale de régénération provient essentiellement de la forte présence d'espèces comme Boscia senegalensis, Acacia ataxacantha, Maytenus senegalensis qui occupent plus de $70 \%$ de l'effectif jeunes. Sur le plan spécifique, la plus part des espèces présente une faible capacité de régénération. Cette tendance pourrait s'expliquer par des phénomènes d'anthropisation avec un système d'utilisation des terres en essor dans la zone de Dakar (Diop et al., 2018). Ce dernier peut affecter la couverture pédologique conditionnant la redistribution de l'eau engendrant ainsi des conditions pouvant être à l'origine de la disparition de certaines essences forestières. La salinisation des sols notée aussi dans la zone due au déficit pluviométrique de ces dernières années qui a entrainé l'abaissement de la nappe phréatique et l'intrusion des eaux de mer à l'intérieur des terres aurait causée des pertes de biodiversité (Ndiaye et al., 2012). De même, la forte progression des activités agricoles dans la zone entrainant le pompage à outrance de cette nappe phréatique pourrait être à l'origine de la disparition des essences forestières à affinité guinéenne dans ce complexe forestier de Noflaye.

\section{Conclusion}

L'étude conduite dans le complexe forestier de la RSBN a contribué à une meilleure connaissance de la structure et des tendances évolutives de cette flore ligneuse. Elle a permis de recenser 65 espèces ligneuses réparties en 26 familles et 50 genres avec une dominance des essences de la famille des Fabaceae (14 espèces). Cette étude a permis de révéler la forte présence des espèces à affinité soudanienne (39\%). Les espèces guinéennes sont faiblement représentées dans le milieu. En termes de représentativité, les espèces les plus nombreuses sont Lepisanthes senegalensis (19,7\%), Neocarya macrophylla (14,5\%) et Grewia bicolor (13,4\%).

L'analyse des paramètres structuraux a montré que la densité réelle du milieu est de 334,5 ind.ha $^{-1}$. Le taux de régénération montre que la densité des sujets âgés est supérieure à celle des jeunes plants. L'évaluation de la diversité spécifique par les indices de Shannon et d'équitabilité montre de forts atouts qui militent en faveur de la conservation de ce poumon vert. Au terme de cette étude, il apparait clairement qu'il existe un changement dans la composition floristique du milieu avec une dominance des essences à 
affinités soudaniennes et sahéliennes au profit des espèces guinéenne. Ainsi donc la mise en place d'un bon plan d'aménagement et de gestion pourrait permettre de sauvegarder ce complexe forestier urbain.

\section{CONFLIT D'INTERETS}

Les auteurs déclarent qu'il n'y a aucun conflit d'intérêts pour cet article.

\section{CONTRIBUTIONS DES AUTEURS}

JD a collecté, traité et analysé les données puis rédigé l'article. AAC, $\mathrm{NdD}$, MSM, BD et $\mathrm{KN}$ ont contribué à la correction de cet article. MS a assuré la partie cartographie.

\section{REFERENCES}

Abdourhamane H, Morou B, Rabiou H, Mahamane A. 2013. Caractéristiques floristiques, diversité et structure de la végétation ligneuse dans le Centre-Sud du Niger : cas du complexe des forêts classées de Dan kada Dodo-Dan Gado. Int. J. Biol. Chem. Sci., 7(3): 1048-1068. DOI: http://dx.doi.org/10.4314/ijbcs.v7i3.13

Adam J. 1957. Flore et végétation de la "réserve" botanique de Noflaye (environs de Dakar). Bull. /FAN ser. A, 20(3) : 809-868.

ANAT (Agence Nationale de l'Aménagement du Territoir). 2015. Schéma directeur d'aménagement et de développement territorial de la zone Dakar-ThièsMbour. Rapport national, ANAT, Sénégal, $163 \mathrm{p}$.

Angiosperm Phylogeny Group. 2009. An update of the Angiosperm Phylogeny Group classification for the orders and families of flowering plants: APG III. Botanical Journal of the Linnean Society, 161: 105-121.

Arbonnier M. 2002. Arbres, arbustes et lianes des Zones d'Afrique de l'Ouest. CIRADMNHN, France.

Ba AT, Noba K. 2001. Flore et biodiversité végétale au Sénégal. Science et Changements Planétaires/Sécheresse, 12(3) : 149-55.
Bassène C, Mbaye MS, Camara AA, Kane A, Gueye M, Sylla SN, Sambou B, Noba K. 2014. La flore des systèmes agropastoraux de la Basse Casamance (Sénégal): cas de la communauté rurale de Mlomp. Int. J. Biol. Chem. Sci., 8(5): 2258-2273.

DOI: http://dx.doi.org/10.4314/ijbcs.v8i5.28

Berhaut J. 1967. Flore du Sénégal (2ème Ed.). Clairafrique ; Dakar : 485 p.

Berhaut J. 1971, 1975, 1979 et 1979. Flore illustrée du Sénégal (Tome I, II, III, IV, $\mathrm{V}$ et VI). Gouvernement du Sénégal, Dakar.

Blondel J. 1979. Biogéographie Ecologie. Edition Masson, collection d'écologie 15; Paris : $173 \mathrm{p}$. http://www.bibliographie-ornithologiefrancaise.fr/ref_detail.php?ref=03066.

Da Sié J. 2009. Etude des usages et de la régénération d'une plante alimentaire au sud-ouest du Burkina Faso: Crataeva adansonii De. (Capparidaceae).

Dessey N. 2006. Dynamique de la végétation et du climat : étude par télédétection de cinq biomes brésiliens, forêt ombrophile dense et ouverte, cerrados, caatinga et campanhagaúcha. Thèse de doctorat, Université Paris X, Nanterre, 362p.

Diallo A, Guisse A, Faye M, Saradoum G. 2009. Variabilité floristique de la végétation herbacée de la Niaye de Pikine au Sénégal. Rev. Ecol. Terre Vie, 64: 123-133.

Diop A, Sambou H, Diop C, Ntiranyibagira E, Dacosta H, Sambou B. 2018. Dynamique d'occupation du sol des zones humides urbanisées de Dakar (Sénégal) de 1942 à 2014. Vertigo, $\mathbf{1 8}(1)$. https://journals.openedition.org/vertigo/2 0120\#tocto2n12.

Diop RD, Mbaye MS, Bassene C, Diop I, Sarr O, Camara AA, Sy MTA, Noba K. 2019. Végétation ligneuse du conservatoire botanique Michel Adanson de Mbour (Sénégal). Int. J. Biol. Chem. Sci., 13(2): 1064-1081. DOI: https://dx.doi.org/10.4314/ijbcs.v13i2.39

Gning ON. 2008. Caractéristiques des ligneux fourragers dans les parcours communautaires de Khossanto (Kédougou, Sénégal Oriental). Mémoire 
de DEA en Biologie végétale, UCAD, $78 \mathrm{p}$.

Gray JS, McIntyre AD, Stirn J.1992. Manuel des méthodes de recherche sur l'environnement aquatique. Onzième partie. Evaluation biologique de la pollution marine, eu égard en particulier au benthos. FAO, Document technique sur les pêches, $\mathrm{N}^{\circ} 324,53 \mathrm{P}$.

Ilboudo. 1992. Etat et tendance évolutives de la flore et de la végétation de la Réserve Spéciale Botanique de Noflaye (environs de Dakar, Sénégal) : éléments pour un aménagement. Thèse de Doctorat de 3ième Cycle, DCAD, Faculté des Sciences et Techniques, Institut des Sciences de l'Environnement, $107 \mathrm{p}$.

Kozlowski TT. 2002. Physiological ecological of natural regeneration of harvested and disturbed forest stands: implications for forest management. Forest Ecology and Management, 158: 195-221.

Lebrun J-P, Stork AL. 1991. Enumération des Plantes à Fleurs d'Afrique Tropicale, Vol. I : Généralités et Annonaceae à Poaceae. Edition Conservatoire et Jardin botaniques de la Ville de Génève; Suisse : p249.

Lebrun J-P, Stork AL. 1992. Enumération des Plantes à Fleurs d'Afrique Tropicale Vol. II: Chrysobalanaceae à Apiaceae. Edition des Conservatoires et Jardin botaniques de la Ville de Génève; Suisse : p257.

Lebrun J-P, Stork AL. 1995. Enumération des Plantes à Fleurs d'Afrique Tropicale Vol. III - Monocotylédones: Limnocharitaceae à Poaceae. Edition des Conservatoire et Jardin botaniques de la Ville de Génève; Suisse : p341.

Lebrun J-P, Stork AL. 1997. Enumération des Plantes à Fleurs d'Afrique Tropicale Vol. IV - Gamopétales : Clethraceae à Lamiaceae. Edition des Conservatoire et Jardin botaniques de la Ville de Génève ; Suisse : p712.

MEPN (Ministère de l'Environnement et de la Protection de la Nature). 1997. Rapport National Biodiversité, MEPN.

Ndiaye O, Diallo A, Matty F, Thiaw A, Fall RD, Guisse A. 2012. Caractérisation des sols de la zone des Niayes de Pikine et de Saint Louis (Sénégal). Int. J. Biol. Chem. Sci., 6(1): 519-528. DOI : http://dx.doi.org/10.4314/ijbcs.v6i1.46

Ngom D, Fall T, Sarr O, Diatta S, Akpo LE. 2013. Caractéristiques écologiques du peuplement ligneux de la réserve de biosphère du Ferlo (Nord Sénégal). $J$. Appl. Biosci., 2013 : 16p. DOI : https://www.researchgate.net/publication 1272326754 .

Osseni AA, Sinsin AB, Teka O. 2014. Facteurs de contrôle de la diversité des plantations d'alignements dans la ville de Porto-Novo au Bénin. Afrique SCIENCE, 10(4) : 200-208.

Sambou B. 2004. Evaluation de l'état, de la dynamique et des tendances évolutives de la flore et de la végétation ligneuses dans les domaines soudanien et subguinéen au Sénégal. Thèse de doctorat, Université Cheikh Anta Diop, 248p.

Sarr. 2018. Caractérisation et identification des plantes grimpantes spontanées de la zone des Niayes de Dakar (Sénégal).s Mémoire de master, Université Cheikh Anta Diop, Dakar, p. 101.

Shannon CE, Weaver W. 1949. The Mathematical Theory of Communication. Press Urbana of Univ. Illionois; $117 \mathrm{p}$.

Tremblay FM, Bergeron Y, Lalond D, Maufette Y. 2002. The sexual effects of potential reproduction and seedling recruitment on the maintenance of red maple (Acer rubrum L.) populations at the northern limit of the species range. Journal of Biogeography, 29: 365-373. DOI:

https://onlinelibrary.wiley.com/doi/abs/.. . /j.1365-2699.2002.00665.

Wouters P, Notelaers V. 1999. L'espace entre les arbres et la densité des peuplements. Sylva Belgica, 106(21): 4. DOI: http://agris.fao.org/agrissearch/search.do ?recordID=BE2000000238

Yeo H, Jang K, Skabardonis A, Seungmo Kang S. 2013. Impact of traffic states on freeway crash involvement rates. Accident Analysis and Prevention, 50 (2013):

713-723. www.elsevier.com/locate/aap. 